Highlighted Version

\title{
Processing and characterization of piezoelectric 0-3 PZT/LCT/PA composites
}

\author{
I Babu ${ }^{1}$, A A van den Ende ${ }^{2-3}$ and G de With ${ }^{1}$
}

${ }^{1}$ Laboratory of Materials and Interface Chemistry, Department of Chemical Engineering and Chemistry, Eindhoven University of Technology, P.O. Box 513, 5600 MB Eindhoven, The Netherlands.

${ }^{2}$ TNO Science and Industry, Materials Performance Department, P.O. Box 6235, 5600 HE Eindhoven, The Netherlands.

${ }^{3}$ Novel Aerospace Materials Group, Faculty of Aerospace Engineering, Delft University of Technology, Kluyverweg 1, 2629 HS Delft, The Netherlands.

E-mail: i.babu@tue.nl

\begin{abstract}
PZT/LCT/PA (Lead Zirconate Titanate $\mathrm{Pb}\left(\mathrm{Zr}_{1-\mathrm{x}} \mathrm{Ti}_{\mathrm{x}}\right) \mathrm{O}_{3} /$ Liquid crystalline thermosets/Polyamide) composites of 0-3 connectivity were fabricated by hot-pressing. Commercially available PZT powder was calcined at different temperatures for the optimization of the composite properties. The phase transition during calcination of the powder was studied by X-ray diffraction and the particle size by light scattering and scanning electron microscopy. The relative permittivity $\varepsilon_{\mathrm{r}}$, piezoelectric charge constant $d_{33}$, conductivity $\sigma$ and elastic modulus $E$ of the composites were found to increase with increasing ceramic volume fraction $\varphi$. The obtained $d_{33}$ and $g_{33}$ values of this newly developed PZT/LCT/PA composite with 50 volume percent PZT using a low poling voltage of $60 \mathrm{kV} / \mathrm{cm}$ and poling time of 30 minutes are $42 \mathrm{pC} / \mathrm{N}$ and $65 \mathrm{mVm} / \mathrm{N}$, respectively, which are high values for this volume fraction in comparison with the other 0-3 composites reported. Good agreement was found between the experimental data of relative permittivity and piezoelectric constants with several theoretical models (Jayasundere, Yamada and Lichtenecker) of 0-3 composites. In order to assess the correlation of the experimental data with the theoretical models, the experimental data obtained from PZT/PA composites were also included.
\end{abstract}


Highlighted Version

\section{Introduction}

Sensors and actuators based on piezoelectric ceramic-polymer composites, so-called smart materials, offer a high potential for high tech systems. These composite materials provide superior overall performance over conventional pure ceramics in having good elastic compliance while maintaining good durability. Usually they are optimized for special applications and the demand for these materials has led to extensive research during the past three decades [1-4]. One of the most used piezoceramics in these types of composites is lead zirconate titanate $\mathrm{Pb}\left(\mathrm{Zr}_{1-\mathrm{x}} \mathrm{Ti}_{\mathrm{x}}\right) \mathrm{O}_{3}(\mathrm{PZT})$ which is a typical piezoelectric material having the perovskite crystal structure. PZT shows excellent electromechanical piezoelectric properties at the morphotropic phase boundary due to the coexistence of the tetragonal and rhombohedral phases and its properties are influenced by the variation in composition [5].

The types and number of phases, composition, and connectivity of the individual phases determine the properties of the composites. Newnham et al. [6] introduced the concept of 0-3 connectivity (a three dimensionally-connected polymer matrix filled with ceramic particles) for the classification of composites. Polymer composites with 0-3 connectivity have several advantages over other types of composites: their ease of production, their ability for the properties to be tailored by varying the volume fraction of the ceramic inclusions and the ease of obtaining different sizes and shapes.

Recently, a number of articles were published on 0-3 composites showing an increased demand on this type of composites [7-10]. The recently developed PZT/ liquid crystalline thermosets (LCT) [11] composites by van den Ende et al. [12] showed excellent high temperature stability. However, although these composites are more flexible than PZT ceramics, they are rather brittle, limiting their potential applications. To overcome this limitation and be able to realize fairly flexible composites with high permittivity and piezoelectric charge constant, we developed a new series of 0-3 piezoelectric composites (PZT/LCT/PA) by incorporating PZT5A4 into a matrix of LCT and polyamide (PA11).

In this paper we report on the processing and characterization of these new 0-3 piezoelectric composites. Hot-pressing was utilized for the fabrication and the effect of the volume fraction of PZT on the composite properties was studied. For comparison also PZT/PA composites were studied. A comparison of the experimental results for relative permittivity $\varepsilon_{\mathrm{r}}$, piezoelectric charge constant $d_{33}$, piezoelectric voltage coefficient $g_{33}$ obtained for these composites with several theoretical models were made. 
Highlighted Version

\section{Experimental}

\subsection{Materials}

The PZT powder used in this research is a half-product of the commercial PZT ceramic PZT5A4 (Morgan Electro Ceramics, Ruabon UK), a soft PZT with $1 \mathrm{~mol} \% \mathrm{Nb}$ added as dopant. The LCT polymer used are phenylethynyl end-capped oligomers based on 6-hydroxy-2-naphthoic acid (HNA) and 4-hydroxybenzoic acid (HBA) $\left(M_{\mathrm{n}}=9000 \mathrm{~g} \mathrm{~mol}^{-1}\right.$, HBA/HNA ratio of 73/27), obtained from TU Delft. The amide was PA11, obtained from Aldrich Chemical Company $\left(T_{\mathrm{g}}=46{ }^{\circ} \mathrm{C}, T_{\mathrm{m}}=198{ }^{\circ} \mathrm{C}\right)$. The PZT powder was calcined at different temperatures (Table 1) with a heating rate of $3{ }^{\circ} \mathrm{C} / \mathrm{min}$ and a 60 minute hold at the required temperature and cooling to room temperature with a temperature ramp of $3{ }^{\circ} \mathrm{C} / \mathrm{min}$. Per calcination temperature 20 gram PZT powder was used in an alumina crucible covered with an alumina lid.

\subsection{Fabrication of composites}

Two types of composites were fabricated. PZT/PA composites were made in order to optimize the calcination temperature and to correlate the theoretical results with PZT/LCT/PA composites. PZT/LCT/PA composites were made with the optimized PZT. Temperatures in the range of 800 to 1300 ${ }^{\circ} \mathrm{C}$ were utilized for optimization.

In order to optimize the calcination temperature, PZT/PA composites were fabricated with 40 volume percent calcined PZT at different temperatures and 60 volume percent PA11. For the correlation of the results with PZT/LCT/PA composites, PZT/PA composites were fabricated with five different volume percent of PZT. The raw materials were initially mixed by hand with a spatula at room temperature and further ball milled for 60 minutes at $800 \mathrm{rpm}$. Composites with specific dimensions of $14 \mathrm{~mm}$ in diameter and 280-300 $\mu \mathrm{m}$ thickness were fabricated by hot-pressing with an applied force of $90 \mathrm{kN}$.

Fig. 1 shows the various PZT/LCT/PA composites fabricated. The corresponding volume percent of PZT and LCT were premixed in an aluminum boat by hand with spatula. This aluminum boat was placed on a hot plate at $285^{\circ} \mathrm{C}$ and thoroughly mixed with a glass rod until all the LCT was molten. After reaching room temperature, the PA powder was added and mixed well. The above mixture was powdered with a pestle and mortar and thereafter subjected to ball-milling for 60 minutes at $800 \mathrm{rpm}$. Composites with specific dimensions of $14 \mathrm{~mm}$ in diameter and 300-375 $\mu \mathrm{m}$ thickness were fabricated by hot-pressing with an applied force of $90 \mathrm{kN}$. 
The LCT was allowed to melt at $280{ }^{\circ} \mathrm{C}$ for $30 \mathrm{~min}$ and after that the temperature was increased to $320{ }^{\circ} \mathrm{C}$ and kept at that temperature for $30 \mathrm{~min}$ for curing the LCT. Circular gold electrodes of thickness $300 \mathrm{~nm}$ and an area of $7.85 \times 10^{-5} \mathrm{~m}^{2}$ are sputtered on both sides of the composites using an Edwards sputter coater (model S150B). The poling of the electroded sample is performed by applying an electric field of 60 $\mathrm{kV} / \mathrm{cm}$ with a Heinziger $10 \mathrm{kV}$ high voltage generator for $30 \mathrm{~min}$ at $100{ }^{\circ} \mathrm{C}(\mathrm{PZT} / \mathrm{PA})$ and $120{ }^{\circ} \mathrm{C}$ (PZT/LCT/PA) in a silicone oil bath to ensure uniform heating. The electric field was kept on while cooling to room temperature.

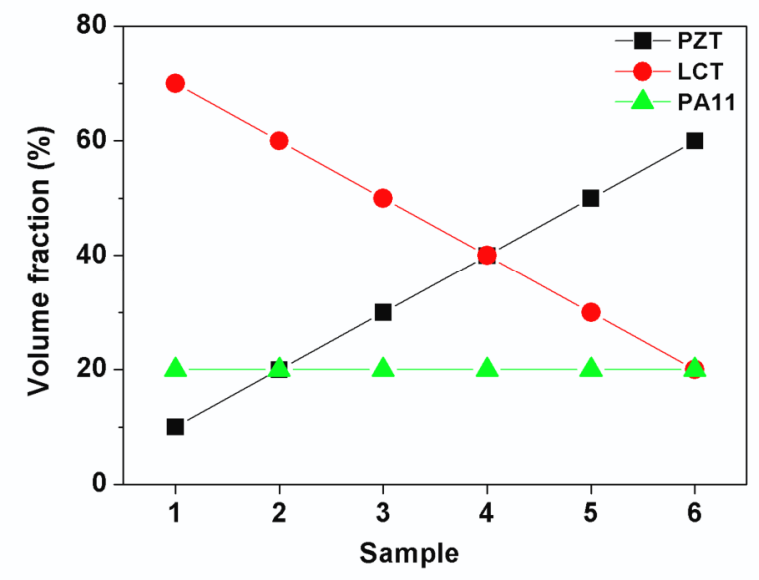

Figure 1. Volume fraction of the PZT/LCT/PA composites fabricated with PZT calcined at $1100{ }^{\circ} \mathrm{C}$.

\subsection{Measurements}

The phase identification was done at room temperature with an X-ray diffractometer (Rigaku) with $\mathrm{CuK} \alpha$ radiation of wavelength $0.15418 \mathrm{~nm}$. The diffraction spectra were recorded in the $2 \theta$ range of $10-80^{\circ}$ with a step size of $0.01^{\circ}$ and a scanning speed of $0.4 \%$ minute. The microstructure of the calcined PZT powder and the composites were examined by SEM (FEI, Quanta 3D FEG). The particle size of the calcined powders was determined by light scattering (Beckman Coulter LS230) and also by SEM. The aspect ratio of the particles was estimated by Image J software [15] on the SEM pictures of the calcined and milled powder.

In order to calculate the ac conductivity $\sigma$, relative permittivity $\mathcal{E}_{\mathrm{r}}$ and the loss tangent $\tan \delta$ of the composites, impedance data were collected by an impedance analyzer (EG\&G Princeton Applied Research, Model 1025) coupled with a potentiostat (Potentiostat/Galvanostat, Model 283) at room temperature in a frequency range of $50 \mathrm{~Hz}-5 \mathrm{MHz}$. The dc conductivity (Table 2) is measured as 
follows. The electrical current was provided by a source measure unit Keithley 237 while the voltage was measured by an electrometer Keithley 6517A. The piezoelectric charge constant $d_{33}$ was measured with a $d_{33}$ meter (Piezotest, PM300) at a fixed frequency of $110 \mathrm{~Hz}$. The piezoelectric voltage coefficient $g_{33}$ was calculated according to

$$
g_{33}=d_{33} / \varepsilon_{0} \varepsilon_{\mathrm{r}}
$$

where $d_{33}$ is the piezoelectric charge constant in $\mathrm{pC} / \mathrm{N}, \varepsilon_{\mathrm{o}}$ is the permittivity of free space $\left(8.85 \times 10^{-12} \mathrm{~F} / \mathrm{m}\right)$ and $\varepsilon_{\mathrm{r}}$ is the relative permittivity of the composite (for this case taken at $110 \mathrm{~Hz}$ ). The elastic moduli of the composites were tested in three-point bending in static mode on a TA Instruments Q800 series DMA at room temperature. The dimensions of the specimens tested were $20 \mathrm{~mm}$ x $10 \mathrm{~mm}$ x $2 \mathrm{~mm}$ and for each composition two specimens were measured.

\subsection{Theory}

Various theoretical models have been proposed for the permittivity and piezoelectric properties of the $0-3$ composites. Some of the mostly used analytic expressions are briefly discussed here. For a brief review we refer to [20] while [24] provides an extensive discussion.

One of the first, if not the first, model for understanding the dielectric behavior of composites, still widely used, was given in 1904 by Maxwell Garnett [24]. In his model spherical inclusions are embedded in a polymer matrix without any kind of interaction resulting in

$$
\varepsilon=\varepsilon_{\mathrm{p}}\left\{1+\left[3 \varphi_{\mathrm{c}}\left(\varepsilon_{\mathrm{c}}-\varepsilon_{\mathrm{p}} / \varepsilon_{\mathrm{c}}+2 \varepsilon_{\mathrm{c}}\right)\right] /\left[1-\varphi_{\mathrm{c}}\left(\varepsilon_{\mathrm{c}}-\varepsilon_{\mathrm{p}} / \varepsilon_{\mathrm{c}}+2 \varepsilon_{\mathrm{c}}\right)\right]\right\}
$$

where $\varphi_{\mathrm{c}}$ is the volume fraction of the inclusions and $\varepsilon, \varepsilon_{\mathrm{c}}$ and $\varepsilon_{\mathrm{p}}$ are the relative permittivity of the composite, ceramic particles and matrix, respectively. Lichtenecker provided in 1923 a rule of mixtures, also still widely used, that reads

$$
\varepsilon=\varepsilon_{\mathrm{c}}^{\varphi_{\mathrm{c}}} \varepsilon_{\mathrm{p}}^{1-\varphi_{\mathrm{c}}}
$$

Although initially largely empirical, in 1998 Zakri et al. [26] provided a theoretical underpinning of this rule.

In 1982 Yamada et al. [18] proposed a model to explain the behavior of the permittivity, piezoelectric constant and elastic constant of a composite in which ellipsoidal particles are dispersed in a continuous 
medium aligned along the electric field. Their model shows excellent agreement with experimental data of PVDF-PZT composites. Their final equations read

$$
\begin{aligned}
& \varepsilon=\varepsilon_{\mathrm{p}}\left[1+\frac{n \varphi_{\mathrm{c}}\left(\varepsilon_{\mathrm{c}}-\varepsilon_{\mathrm{p}}\right)}{n \varepsilon_{\mathrm{p}}+\left(\varepsilon_{\mathrm{c}}-\varepsilon_{\mathrm{p}}\right)\left(1-\varphi_{\mathrm{c}}\right)}\right] \\
& d=\alpha_{\mathrm{p}} \varphi_{\mathrm{c}} G d_{\mathrm{c}} \quad \text { and } \\
& E=E_{\mathrm{p}}\left[1+\frac{\varphi_{\mathrm{c}}\left(E_{\mathrm{c}}-E_{\mathrm{p}}\right)}{E_{\mathrm{p}}+n^{\prime}\left(E_{\mathrm{c}}-E_{\mathrm{p}}\right)\left(1-\varphi_{\mathrm{c}}\right)}\right] \text { with } n^{\prime}=\frac{1+v_{\mathrm{p}}}{3\left(1-v_{\mathrm{p}}\right)}
\end{aligned}
$$

where $n=4 \pi / m$ is the parameter attributed to the shape of the ellipsoidal particles, $\varphi_{\mathrm{c}}$ is the volume fraction of the inclusions and $\varepsilon, \varepsilon_{\mathrm{c}}$ and $\varepsilon_{\mathrm{p}}$ are the relative permittivity of the composite, ceramic and the matrix, respectively. Further, $\alpha$ is the poling ratio, $G=n \varepsilon /\left[n \varepsilon+\left(\varepsilon_{c}-\varepsilon\right)\right]$ is the local field coefficient and $d_{\mathrm{c}}$ is the piezoelectric constant of the piezoceramic while $d$ is the piezoelectric constant of the composite. Finally the elastic modulus $E$ contains, apart from the above mentioned quantities, a factor $n^{\prime}$ directly calculated from Poisson's ratio $v_{\mathrm{p}}$ of the matrix. The condition that the particles are considered to be oriented ellipsoids might seem to be a significant restriction but it has been shown [26] that composites with an arbitrary distribution of ellipsoids with respect to the electric field direction can be transformed in to an equivalent composite with ellipsoids aligned along the electric field direction but with different aspect ratio's for the ellipsoids. This largely removes the restriction mentioned, although the interpretation in micro structural terms becomes more complex. Since their model also provides an expression for the elastic modulus, we have chosen this model mainly to analyze our experimental results.

Another model relatively simple model for the permittivity was provided by Jayasundere et al. [21]. The final expression reading

$$
\varepsilon=\frac{\varepsilon_{\mathrm{p}} \varphi_{\mathrm{p}}+\varepsilon_{\mathrm{c}} \varphi_{\mathrm{c}}\left[3 \varepsilon_{\mathrm{p}} /\left(\varepsilon_{\mathrm{c}}+2 \varepsilon_{\mathrm{p}}\right)\right]\left[1+3 \varphi_{\mathrm{c}}\left(\varepsilon_{\mathrm{c}}-\varepsilon_{\mathrm{p}}\right) /\left(\varepsilon_{\mathrm{c}}+2 \varepsilon_{\mathrm{p}}\right)\right]}{\varphi_{\mathrm{p}}+3 \varphi_{\mathrm{c}} \varepsilon_{\mathrm{p}} /\left(\varepsilon_{\mathrm{c}}+2 \varepsilon_{\mathrm{p}}\right)\left[1+3 \varphi_{\mathrm{p}}\left(\varepsilon_{\mathrm{c}}-\varepsilon_{\mathrm{p}}\right) /\left(\varepsilon_{\mathrm{c}}+2 \varepsilon_{\mathrm{p}}\right)\right.}
$$

is a modification of the expression for a composite dielectric constant of a sphere with permittivity $\mathcal{E}_{\mathrm{c}}$ in a matrix with permittivity $\varepsilon_{\mathrm{p}}$ by including interactions between neighboring spheres. The comparison with experimental data appeared to be excellent. Hence this model was applied as well. 
Many other models resulting in analytical expressions have been proposed. We mention here only the models by Furukawa et al. [19], Bruggeman et al. [8], Maxwell-Wagner [22], Bhimasankaram et al. [20] and Wong et al. [27]. Most models deal only with part of the full piezoelectric problem and only partially combined solutions were given. E.g. based on the Bruggeman method [29, 30], taking permittivity and conductivity into account, or based on the Marutake method [31], taking permittivity piezoelectric coefficients and elastic compliance into account. The latter already requires numerical solution. Recently complete numerical solutions to the fully coupled piezoelectric equations for ellipsoidal inclusions of the same orientation have been provided [28] predicting giant piezoelectric and dielectric enhancement. No experimental evidence for this effect was presented though while a significant conductivity is required rendering the options for practical applications probably less useful.

\section{Results and discussion}

\subsection{Optimization of PZT}

\subsubsection{X-ray diffraction}

The XRD patterns of PZT5A4 powder before and after calcination at different temperatures (800 to 1300 ${ }^{\circ} \mathrm{C}$ ) are shown in figure 2. The X-ray diffraction pattern of the PZT5A4 before calcination shows the coexistence of both rhombohedral (200)R and tetragonal phases [(002)T, (200)T] together with the presence of a pyrochlore phase. Calcination resulted in the disappearance of the rhombohedral perovskite structure and in the formation of peak splitting, indicating an increase of tetragonal distortion. The phenomenon of peak splitting and peaks shifting to higher angle with increasing calcination temperature was also reported in previous studies [13, 14].

As the calcination temperature increases, the intensity of the pyrochlore phase peaks decrease while the intensity of the tetragonal perovskite peaks increase. This indicates the transformation to almost single phase tetragonal PZT. The PZT calcined at $1100{ }^{\circ} \mathrm{C}$ shows a maximum intensity of the tetragonal perovskite peaks at (200)T and (211)T which indicates that at this temperature the material has become (XRD) single phase. From $1150{ }^{\circ} \mathrm{C}$ onwards, the peak height decreases as a result of lead loss and also the 2:1 ratio becomes more like 1:1. Zhang et al. reported that the shrinkage of the lattice is believed to result from loss of lead that creates some vacancies in the PZT lattice [14]. The optimal calcination temperature was found to be $1100{ }^{\circ} \mathrm{C}$ and PZT5A4 calcined at this temperature was used for composite fabrication. 


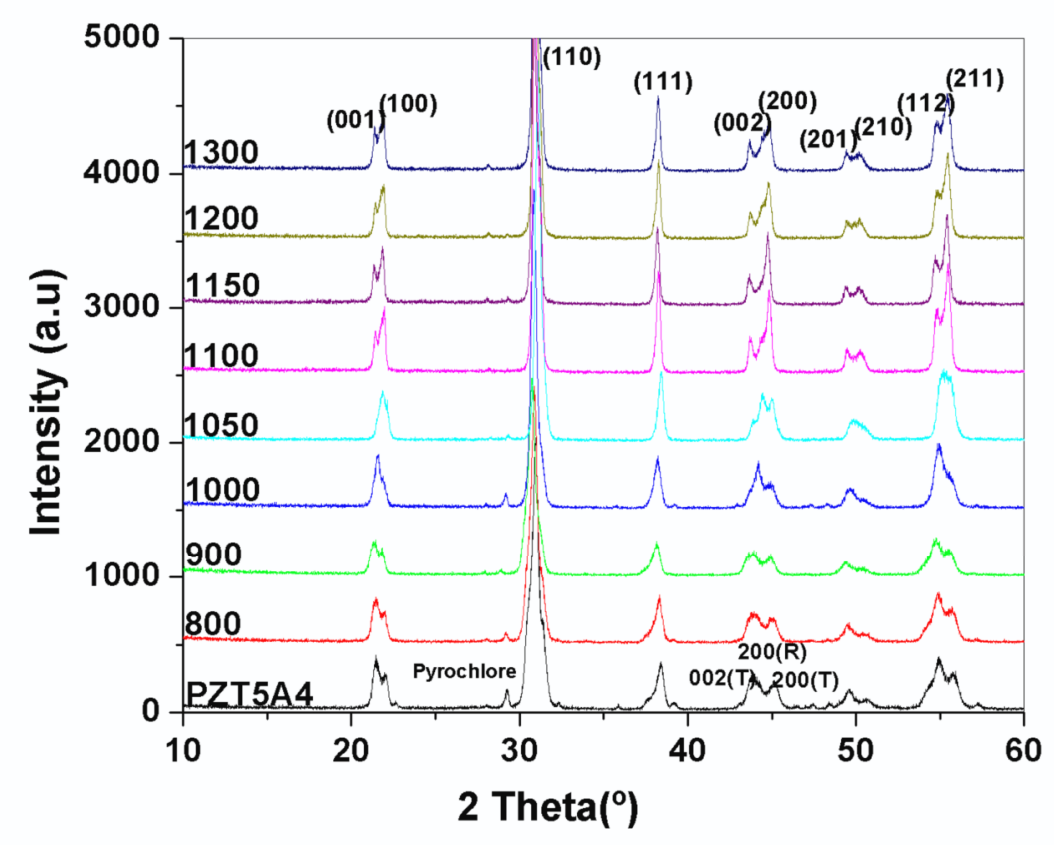

Figure 2. XRD patterns of PZT5A4 at different calcination temperatures.

\subsubsection{Particle size analysis}

The size of the particles was analyzed by both light scattering and by SEM. Table 1 illustrates the influence of different calcination temperatures on the particle size. The particles were also examined by SEM to get an average size of the individual particles. Comparing the particle size obtained from light scattering with SEM images indicates that the particles are agglomerates.

Figure 3 shows the SEM micrographs of the calcined PZT5A4 at different temperatures. From these micrographs it is observed that, as the calcination temperature increases, the grain size gradually increases, which implies that the particles sinter together during the calcination process. The approximate primary particle size in PZT5A4 calcined at $1000{ }^{\circ} \mathrm{C}$ is less than $600 \mathrm{~nm}$, at $1100{ }^{\circ} \mathrm{C}$ the average size is about $1.0 \mu \mathrm{m}$ and as the calcination temperature reaches to $1200{ }^{\circ} \mathrm{C}$, the size of the primary particles becomes 2 to $2.5 \mu \mathrm{m}$. The number of particles per agglomerate can be estimated as about $\left(d_{50} / d_{\text {SEM }}\right)^{3}$. For $1100{ }^{\circ} \mathrm{C}$ this yields $(2.8 / 1.0)^{3} \cong 22$ primary particles which appears to be not an unreasonable number. 
Highlighted Version

Table 1. Particle sizes of calcined PZT5A4 as determined by light scattering.

\begin{tabular}{lccc}
\hline Calcination Temperature $\left({ }^{\circ} \mathrm{C}\right)$ & $d_{10}(\mu \mathrm{m})$ & $d_{50}(\mu \mathrm{m})$ & $d_{90}(\mu \mathrm{m})$ \\
\hline PZT5A4 powder & 0.23 & 1.47 & 3.4 \\
800 & 0.88 & 1.78 & 3.74 \\
900 & 1.00 & 1.94 & 3.98 \\
1000 & 1.37 & 2.39 & 5.23 \\
1050 & 1.25 & 2.16 & 4.17 \\
1100 & 1.38 & 2.81 & 9.08 \\
1150 & 1.51 & 3.56 & 11.07 \\
1200 & 1.31 & 3.61 & 21.09 \\
1300 & 0.71 & 3.61 & 17.39 \\
\hline
\end{tabular}
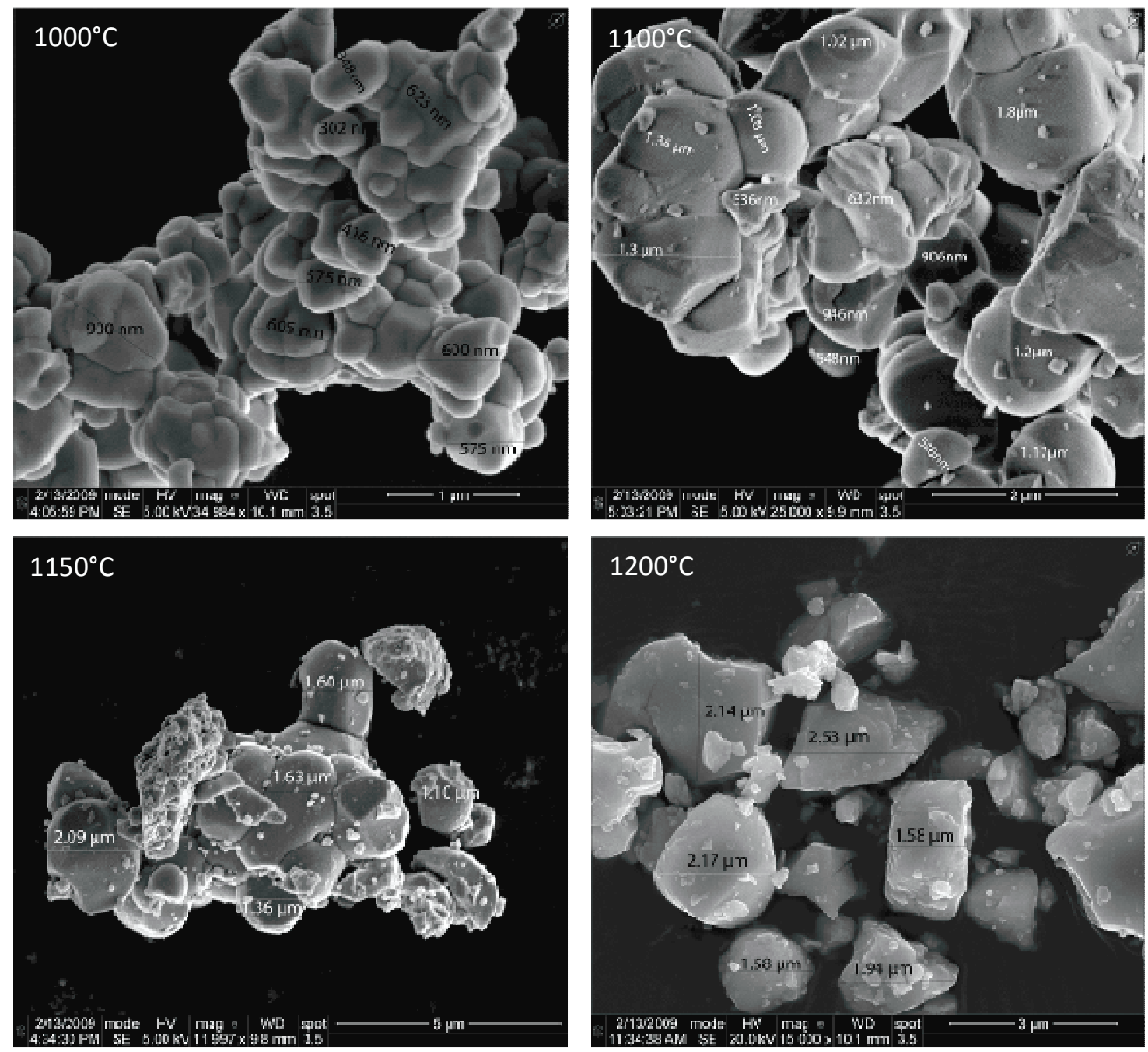

Figure 3. SEM micrographs of the PZT5A4 calcined at different temperatures. 


\subsubsection{Impedance data}

For the fabricated PZT/PA composites, the ac conductivity $\sigma$, relative permittivity $\varepsilon_{\mathrm{r}}$, loss tangent $\tan \delta$, piezoelectric charge constant $d_{33}$ and piezoelectric voltage constant $g_{33}$ were determined. Figure 4 (a) and (b) show the dependence of $\sigma, \varepsilon_{\mathrm{r}}$ and $\tan \delta$ as a function of $\log$ frequency with increasing calcination temperature for the PZT/PA composites. From figure 4 (a) it is observed that the ac conductivity is the same for all calcination temperatures and equal to the value for the as-received PZT composite. From figure 4 (b) it is observed that the relative permittivity is in the range from 28 to 38 for the PZT calcined at different temperatures ranging from the as-received PZT powder to the one calcined at $1300{ }^{\circ} \mathrm{C}$. The maximum relative permittivity is observed at $1000{ }^{\circ} \mathrm{C}$ and $1100{ }^{\circ} \mathrm{C}$. From $1200{ }^{\circ} \mathrm{C}$, a small decrease in $\varepsilon_{\mathrm{r}}$ is observed which may be due to the loss of lead from PZT. The $\tan \delta$ has a weak dependence on calcination temperature and is about 0.02 to 0.06 neglecting scattered points.
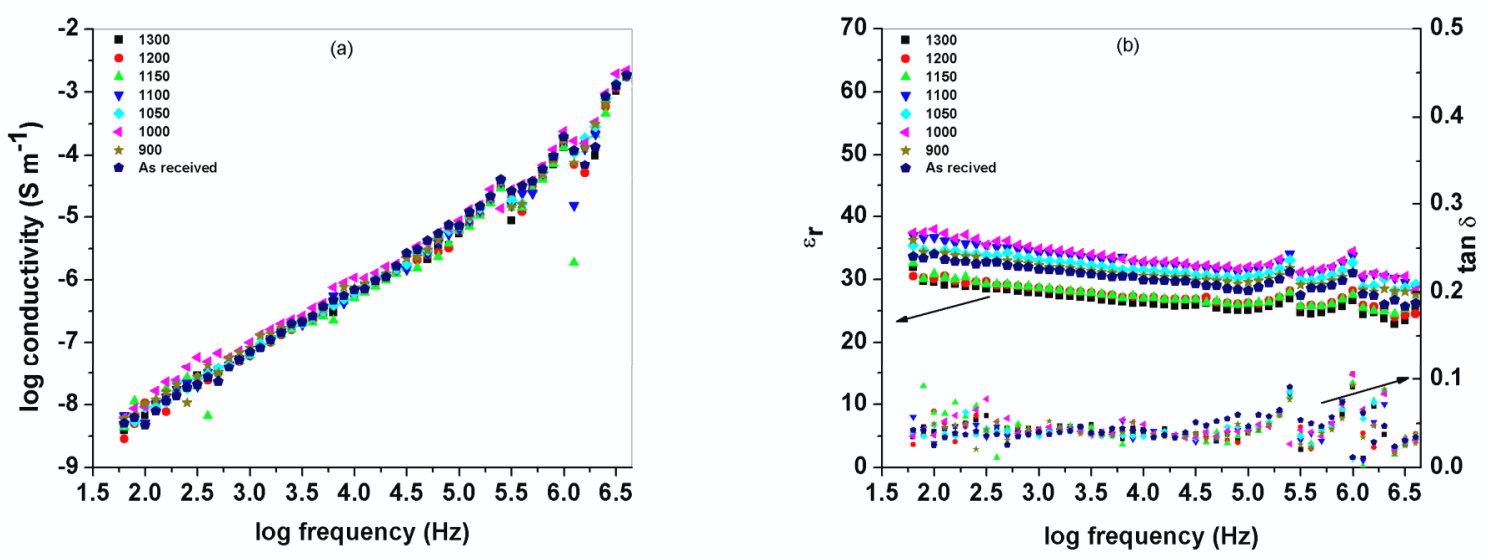

Figure 4 (a) and (b). Dependence of $\sigma, \varepsilon_{\mathrm{r}}$ and $\tan \delta$ as a function of $\log$ frequency with increasing calcination temperature for the PZT/PA composites.

\subsubsection{Piezoelectric charge and voltage constant}

The dependence of $d_{33}$ and $g_{33}$ with increasing calcination temperature is shown in figure. 5. It is observed that the composite using PZT calcined at $1100{ }^{\circ} \mathrm{C}$ shows the maximum value of $d_{33}$ and $g_{33}$. As the calcination temperature increases, a decrease in piezoelectric voltage coefficient is observed. Since $\varepsilon_{\mathrm{r}}$ is nearly constant up to $1150{ }^{\circ} \mathrm{C}$ and thereafter decreases but slightly, the $g_{33}$ behavior mimics the $d_{33}$ behavior closely. This may be due to the decrease in lead content, since the piezoelectric effect is highly dependent on the amount of lead in the PZT. From the above results it is clear that the best quality PZT 
Highlighted Version

powder is obtained with a calcination temperature of $1100{ }^{\circ} \mathrm{C}$, consistent with the $\mathrm{X}$-ray results on the PZT powder.

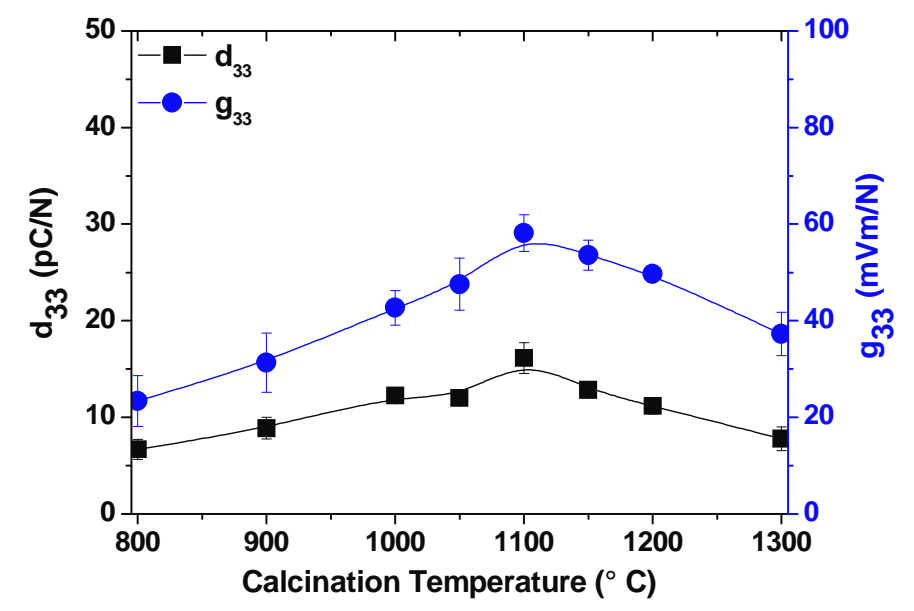

Figure 5. The dependence of $d_{33}$ and $g_{33}$ of the PZT/PA composites on the PZT calcination temperature (lines drawn as guide to the eye).

\subsection{Fabrication of the PZT/LCT/PA composite with optimized PZT}

\subsubsection{Impedance data}

Figure 6 (a) and (b) show the dependence of ac conductivity $\sigma$, permittivity $\varepsilon_{\mathrm{r}}$ and $\tan \delta$ as a function of $\log$ frequency with increasing PZT volume fraction for the PZT/LCT/PA composites. From figure 6 (a) it is observed that, as the volume percentage of PZT increases, the conductivity increases. Also an increase in $\varepsilon_{\mathrm{r}}$ is observed as the volume percentage of PZT increases, due to the increasing contribution of the PZT. Since the particles in the composite are tightly packed with limited agglomeration and porosity, a homogeneous particle distribution results, even for a high volume percentage of PZT, as shown by the SEM images (Figure 11). This probably leads to the comparatively high $\varepsilon_{\mathrm{r}}$ values of the PZT/LCT/PA composite in comparison with those of other composites reported in literature (Table 3). The $\tan \delta$ has a weak dependence on the volume fraction of PZT ranging from 0.01 to 0.06 if the scattered points are not considered. 

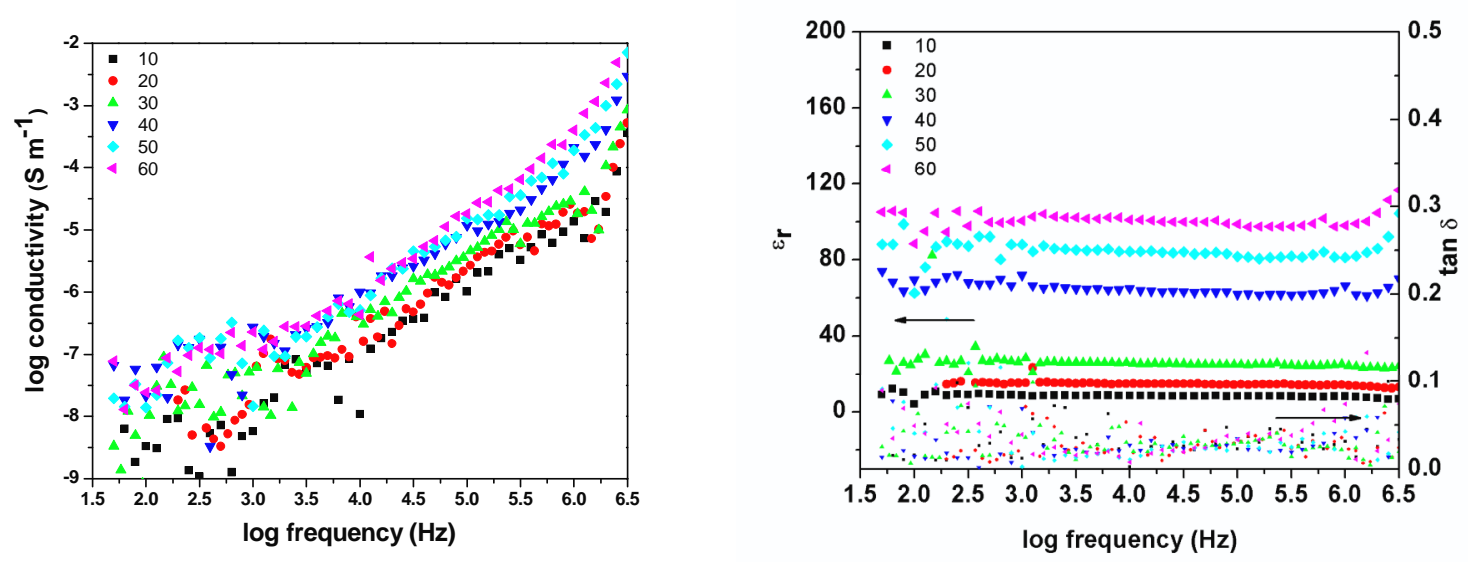

Figure 6 (a) and (b). Dependence of $\sigma, \varepsilon_{\mathrm{r}}$ and $\tan \delta$ as a function of $\log$ frequency with increasing PZT volume fraction for the PZT/LCT/PA composites.

\subsubsection{Piezoelectric charge and voltage constant}

The dependence of the $d_{33}$ and $g_{33}$ values of the PZT/LCT/PA composites with increasing volume fraction of PZT is shown in figure 7. The $d_{33}$ value of the composite shows a continuous increase with increasing volume fraction of PZT, while the $g_{33}$ value shows a maximum at 50 volume percent and then decreases when it reaches 60 volume percent. This decrease of $g_{33}$ is due to the behavior of $d_{33}$ relative to $\varepsilon_{\mathrm{r}}$. A maximum value for $d_{33}$ of $46 \mathrm{C} / \mathrm{N}$ is observed for the 60 volume percent PZT while for $g_{33}$ a maximum value of $65 \mathrm{mVm} / \mathrm{N}$ is obtained, both for the 50 volume percent PZT composite.

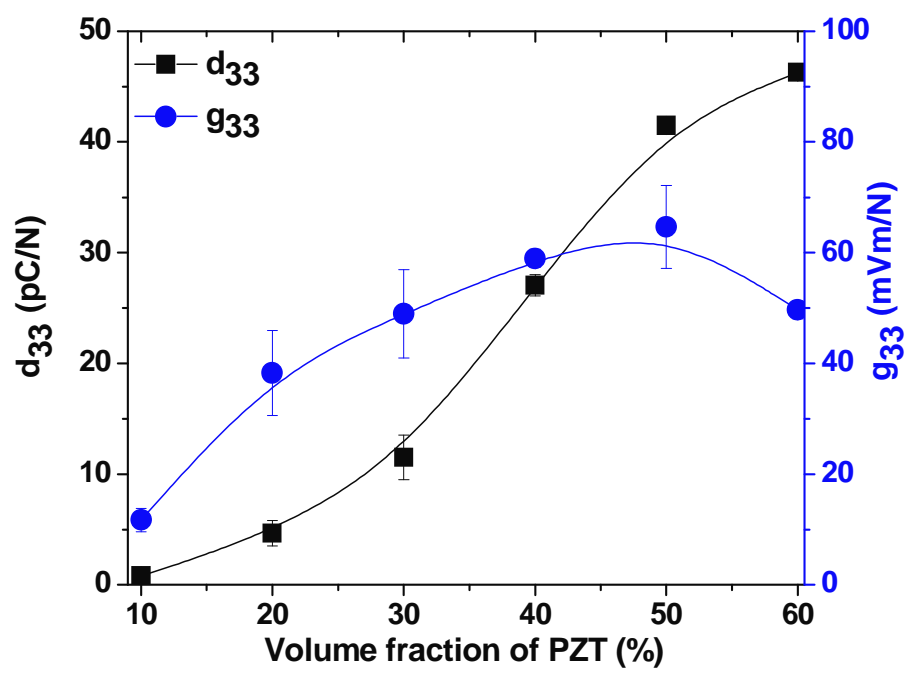

Figure 7. Dependence of the $d_{33}$ and $g_{33}$ of the PZT/LCT/PA composites with increasing volume fraction of PZT (lines drawn as a guide to the eye). 
The data used in the various calculations are given in Table 2. Table 3 provides the optimum data of $\varepsilon_{\mathrm{r}}$, $d_{33}$ and $g_{33}$ obtained for the PZT/LCT/PA and PZT/PA composites and a comparison of different polymer/PZT composites as reported in the literature. The $d_{33}$ and $g_{33}$ values obtained in the present work follows the same trend as reported by Yamada et al. [18], Satish et al. [16] and Venkatragavaraj et al. [9]. Satish et al. reported that the hot-pressing is the most reliable method for the fabrication of $0-3$ composites. Moreover hot-pressed composites are known to posses better piezoelectric properties than solution-cast composites [9].

Table 2: Data of the various components used for the calculations for the composites.

\begin{tabular}{clclll}
\hline Type & $\varepsilon_{\mathrm{r}}$ & $\tan \delta$ & $d_{33}(\mathrm{pC} / \mathrm{N})$ & $E(\mathrm{GPa})$ & $\mathrm{dc} \sigma(\mathrm{S} / \mathrm{m})$ \\
\hline PZT & $1820^{\mathrm{a}}$ & - & 480 & $37^{\mathrm{c}}$ & - \\
LCT & 3.6 & $0.0025^{\mathrm{b}}$ & 0 & 1.0 & $1.22 \cdot 10^{-11}$ \\
PA11 & 3.9 & $<0.035^{\mathrm{b}}$ & $<0.5$ & 0.83 & $3.82 \cdot 10^{-11}$ \\
\hline
\end{tabular}

${ }^{\mathrm{a}}$ Data from Morgan Electroceramics.

${ }^{\mathrm{b}}$ Data measured at $110 \mathrm{~Hz}$.

${ }^{\mathrm{c}}$ Data from [32].

Table 3: Data of $\varepsilon_{\mathrm{r}}, d_{33}$ and $g_{33}$ obtained for the optimum PZT/LCT/PA and PZT/PA composites and comparison with literature data.

\begin{tabular}{llcc}
\hline Type & $\varepsilon_{\mathrm{r}}$ & $d_{33}(\mathrm{pC} / \mathrm{N})$ & $g_{33}(\mathrm{mVm} / \mathrm{N})$ \\
\hline LCT/PA/0.5 PZT (present work) & 73 & 42 & 65 \\
PA/0.5 PZT (present work) & 68 & 28 & 48 \\
LCT/0.4 PZT [11] & 30 & 13 & 48 \\
PVDF/0.7 PZT [16] & 100 & 26 & 30 \\
Epoxy/0.685 PZT [17] & 120 & 50 & 47 \\
PVDF/0.67 PZT [18] & 152 & 48 & 36 \\
PVDF/0.5 PZT (Hot-press) [9] & 95 & 14 & 16 \\
PVDF/0.5 PZT (Solution cast) [9] & 30 & 9 & 36 \\
\hline
\end{tabular}

${ }^{a} 1.5 \%$ carbon black added to the PZT-epoxy mixture.

\subsubsection{Comparison with theoretical models}

In figures 8-10 the experimental data of the PZT/LCT/PA and PZT/PA composites is compared to several models for 0-3 composites, both for dielectric and piezoelectric constants. It is observed that both the composites follow the same trend. In the PZT/LCT/PA, the relative permittivity of the matrix phase was 
Highlighted Version

taken to be the combined relative permittivity of the LCT and PA11 phases and was calculated by Lichtenecker's rule of mixtures [23] given by equation 3. The relative permittivity's of virgin LCT and PA11 materials were found to be 3.6 and 3.9, respectively, and the relative permittivity of the ceramic phase was taken to $\varepsilon_{\mathrm{r}}=1820$ (data Morgan electroceramics, UK). The relative permittivity of the PZT/LCT/PA composite was calculated using Lichtenecker's formula and 2 models for high filler content 0-3 composites, namely Yamada's model [18] and Jayasundere's model [21].
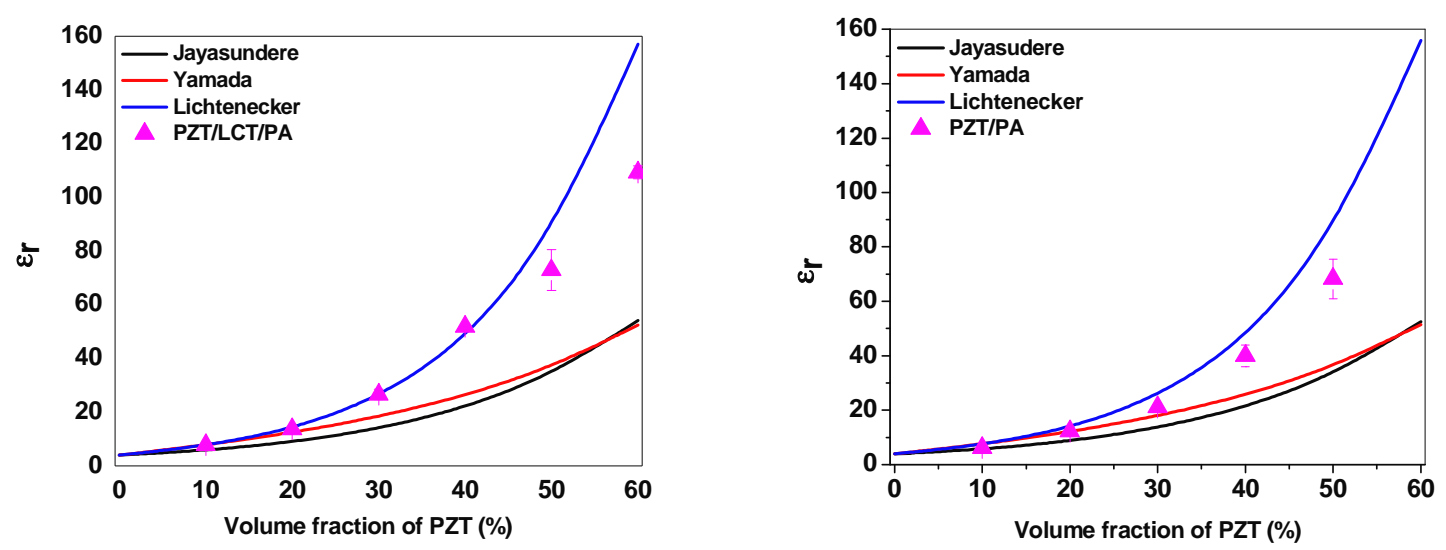

Figure 8. The relative permittivity values of the PZT/LCT/PA and PZT/PA composites as compared to 3 models for $0-3$ composites.

The fit of the equation to the data points of the composites for both these models is plotted in figure 8 . Both Yamada's and Jayasundere's model underestimate the permittivity of the composites, while Lichtenecker's mixing rule correlates well with the measured values up to 50 volume percent PZT for the PZT/LCT/PA composites. The dielectric behavior therefore, seems to satisfy the logarithmic mixing rule for the PZT/LCT/PA composites. The discrepancy at 60 volume percent is probably due to the fact that at this volume fraction processing becomes much more difficult.

The strong increase in relative permittivity with the increase in PZT volume fraction indicates good coupling of the PZT phase with the polymer phases up to high volume percentages. In both composites the discrepancy between the experimental data and theoretical models is higher at higher volume fraction. Two effects probably play a role here. According to theory the dielectric constant increases with increasing ellipticity of the particles [18, 24, 25]. Moreover, at higher volume fraction the composites, no more act as 0-3 but as 1-3 composites and this is another reason for the high relative permittivity.

In figure 9 the $d_{33}$ values are calculated using Yamada's model [18] for high PZT content composites with the $d_{33}$ of the ceramic phase taken to be $d_{33}=480 \mathrm{pC} / \mathrm{N}$ (data Morgan electroceramics, UK). This model 
was originally developed for PZT/PVDF composites and tested to a PZT content of 70 volume percent. Since the predicted values mainly depend on the permittivity of the composite and the volume fraction filler, both composites are represented by the same theoretical line. The model shows good correlation of measured $d_{33}$ values up to 30 volume percent PZT for both composites, while at 40 and 50 volume percent some deviations occur. For 60 volume percent the estimated values are lower than expected, and as indicated, this may be due to the increased processing difficulties. It is observed that the presence of LCT results in an increase in $\varepsilon_{\mathrm{r}}$ and $d_{33}$ values. This may be due to some preferred orientation of the LCT around the PZT particles, leading to increased polarisability.

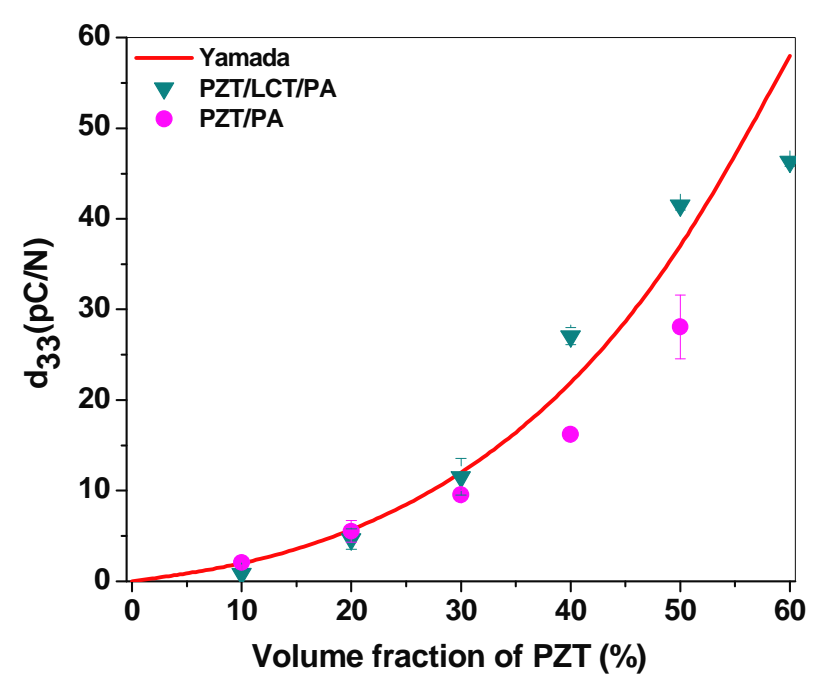

Figure 9. The $d_{33}$ values of PZT/LCT/PA and PZT/PA composites as compared to Yamada's model for piezoelectric composites.

The Yamada model incorporates anisotropy effects of the particles by the parameter $n$. If $n=3$, the particles are spherical while $n>3$ reflects elongated particles. The best fit for $d_{33}$ is obtained with a shape parameter of $n=8.5$, which is similar to the value that Yamada et al. found for their composites. To corroborate this, the aspect ratio for 40 particles was estimated from SEM pictures resulting $1.6 \pm$ 0.2 , which corresponds to a value of $n=4.6$. This factor is lower than the factor of $n=8.5$ obtained from fitting the experimental $d_{33}$ data to the model. This possibly can be explained by increasing particleparticle interactions at high filling fraction in the composites. When calculating piezoelectric constants using Yamada's model, this translates to a higher $n$ value, which is explained by the model as a higher aspect ratio of the particles. In reality, simply more particles are directly connected which leads to a more direct electromechanical coupling between the particles themselves. Moreover, Yamada's model assumes that all particles are oriented along the direction of the electric field. While this is obviously not 
true, it has been shown [16] that a random configuration of ellipsoids can be transformed to an equivalent, completely oriented configuration but with different (effective) aspect ratio's. Hence the aspect ratio as observed microscopically not necessarily agrees with the one determined by dielectric measurements.

The combination of LCT and PA11 increases the poling effectiveness, even at relatively low poling voltages. This is evident when comparing $d_{33}$ results for the composite with a LCT/PA matrix (figure 7) to the composite with PA matrix only (figure 5). The $40 \%$ PZT composite with an LCT/PA matrix has a $d_{33}$ value of 1.7 times the value for composite with a PA11 matrix only. The resulting $g_{33}$ values are calculated using equation 1 . It can be seen in figure 10 that the values calculated using the Lichtenecker model for the relative permittivity and Yamada's model for $d_{33}$ correlate reasonably well with the $g_{33}$ values of the PZT/LCT/PA composites, while values calculated with the relative permittivity predictions of both other models overestimate the $g_{33}$ as a result of the underestimation in relative permittivity.

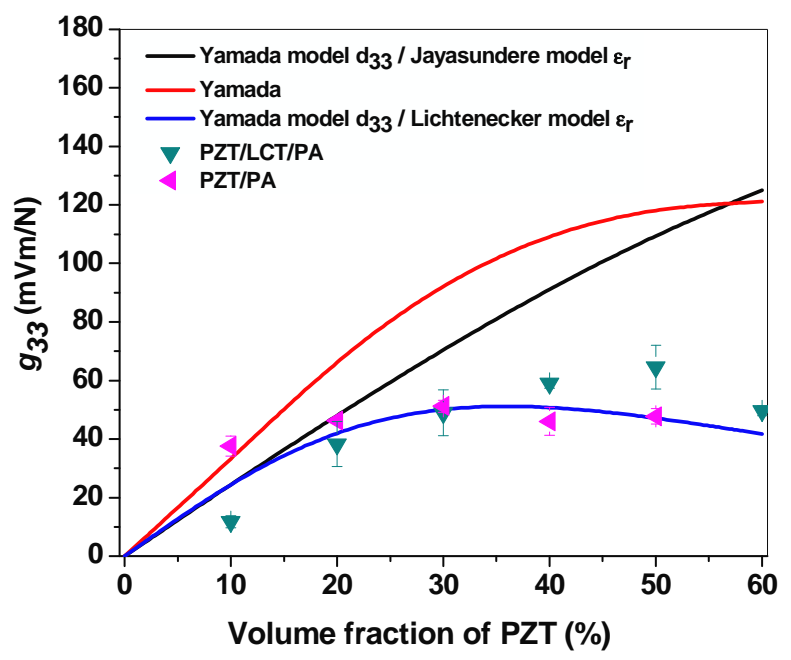

Figure 10. The $g_{33}$ values of the PZT/LCT/PA and PZT/PA composites as compared to 3 models.

\subsubsection{SEM Morphology}

Figure 11 shows the SEM surface morphology of PZT/LCT/PA composites with 20, 30, 40, 50 and 60 volume percent PZT. From these figures it is observed that all the composites have a relative density near to $100 \%$. Moreover, on the scale of tens of micrometers the composites appear to be homogeneous and have a very similar microstructure. From the 60 volume percent PZT image it also becomes clear that the microstructure is also homogeneous at the micrometer scale. The detailed view of structure of the 60 volume percent PZT composite shows the good adhesion of polymer matrix with the ceramic particles. From these and similar pictures we conclude that the PZT particles are homogeneously dispersed in the polymer matrix and are well adhering to the polymer matrix. 
Highlighted Version
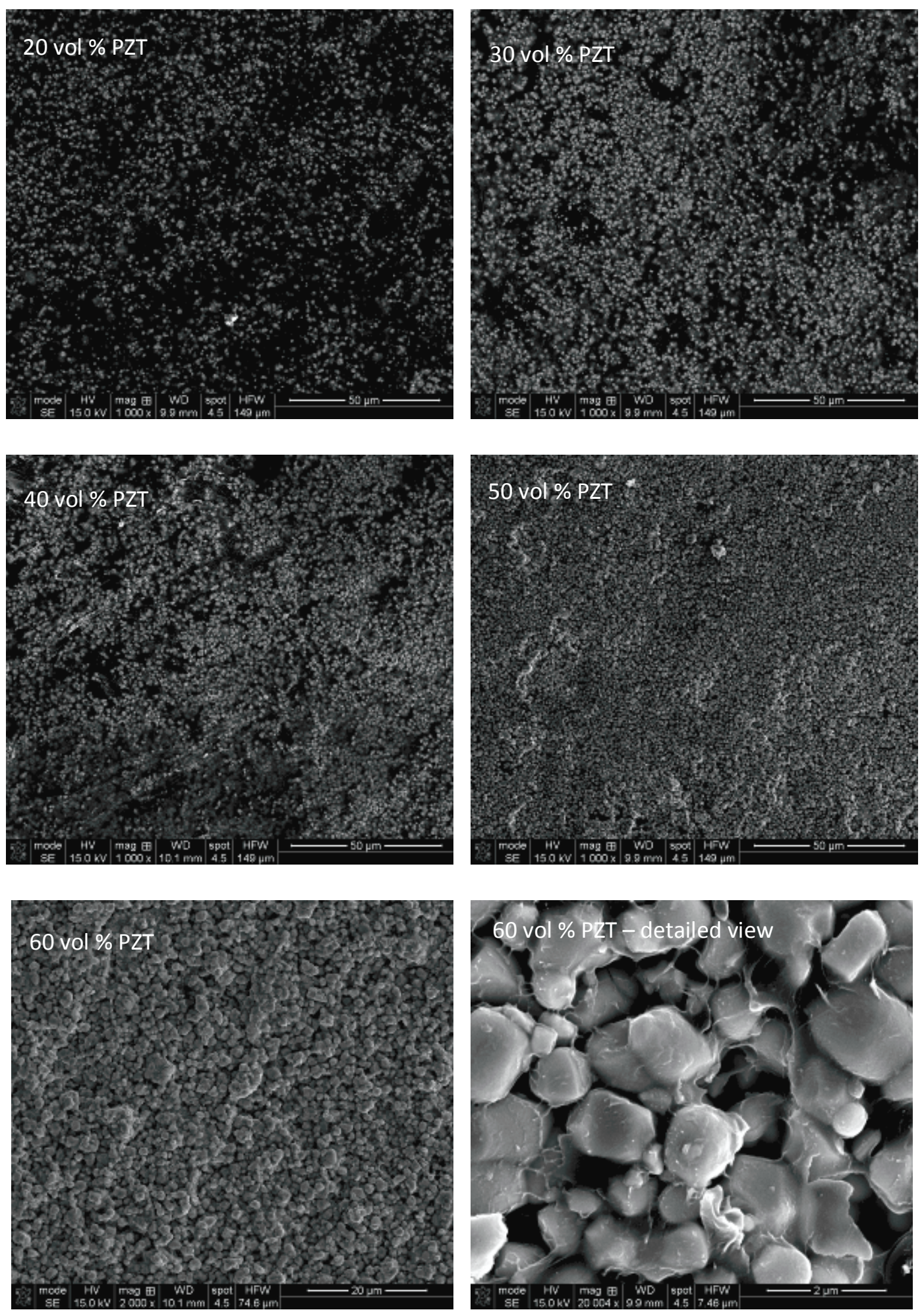

Figure 11. SEM surface morphology of the PZT/LCT/PA composites with 20, 30, 40, 50 and 60 volume percent of PZT. The bright colour represents the PZT and the dark background the polymer. 


\subsubsection{Mechanical properties by DMA}

Figure 12 shows the stress-strain curves of the PZT/LCT/PA and PZT/PA composites including the pure polymer and the ceramic volume fraction up to $40 \%$. The measurements are done at a strain rate of $0.5 \%$ $\min ^{-1}$ at room temperature. The three-point bending mode was utilized for these measurements with a preload force of $0.1 \mathrm{~N}$. The upper limit of the static force of the machine is $18 \mathrm{~N}$ and the measurements stops when the force reaches this point. Young's modulus $E$ is calculated from the slope near the origin.
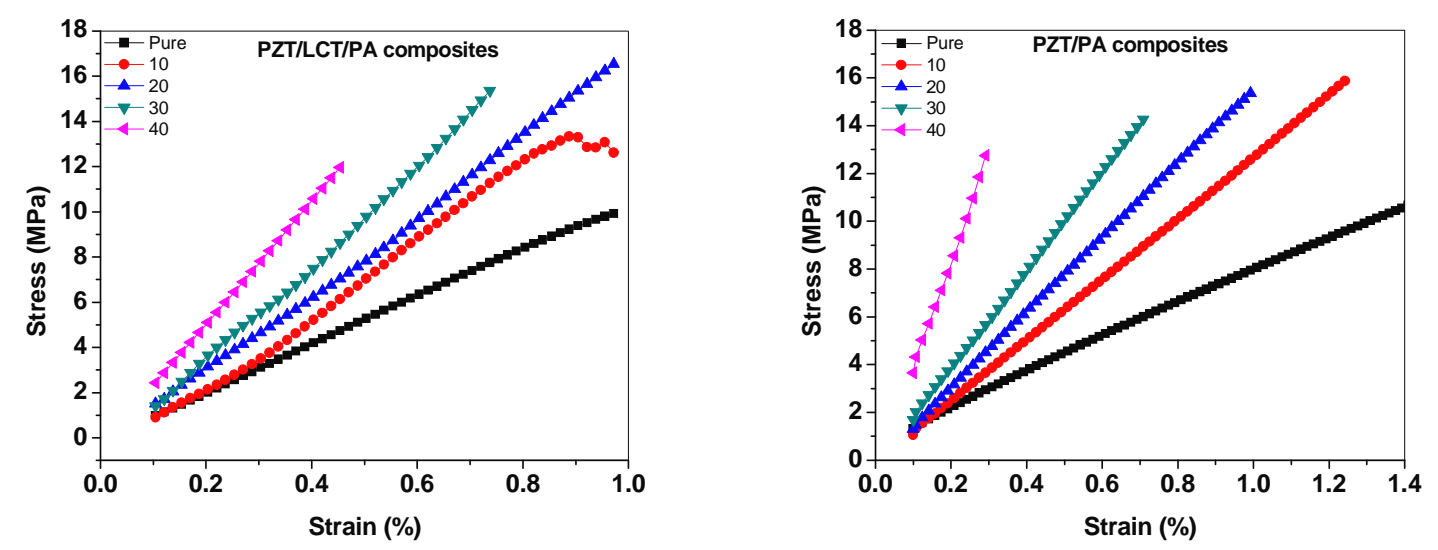

Figure 12. Stress-strain curves of the PZT/LCT/PA and PZT/PA composites at room temperature as measured in three-point bending with DMA.

From the graphs it can be observed that the resulting strain decreases strongly with increasing volume fraction of PZT leading to increasing Young's modulus. Young's modulus ranges from 1050 - $3000 \mathrm{MPa}$ for the PZT/LCT/PA composites and from 850 to $4450 \mathrm{MPa}$ for the PZT/PA composites. The modulus obtained for pure LCT (1.0 GPa) is lower than the value of 3.2 GPa as reported by Knijnenberg et al. [11]. However, since the strain rate in the static mode (as used by us) is about 4 orders of magnitude lower than the one for the dynamic mode (as used by [11]) and the former generally leads to lower Young's modulus values, this difference in measuring modes probably explains the difference in elastic values observed.

The elastic modulus of the composites was also calculated according to Yamada's model (Eq. 6) using $E_{\mathrm{c}}$ $=37 \mathrm{GPa}$ and $v_{\mathrm{p}}=0.35$. While the agreement for the PZT/LCT/PA composites is fair, the agreement for the PZT/PA composites is less good (figure not shown) and we refrain from further discussion. 
Highlighted Version

\section{Conclusions}

A series of new 0-3 PZT/LCT/20\%PA composites was successfully fabricated and characterized. X-ray diffraction indicated that for the PZT powder used, calcination at $1100{ }^{\circ} \mathrm{C}$ shows the maximum intensity for the single phase tetragonal perovskite peaks. The obtained $d_{33}$ and $g_{33}$ values from PZT/PA composites are also the highest for the PZT calcined at $1100{ }^{\circ} \mathrm{C}$, supporting the above conclusion. The experimental results from $\varepsilon_{\mathrm{r}}, d_{33}$ and $g_{33}$ indicates that the new composites have high $\varepsilon_{\mathrm{r}}$, and possess high $d_{33}$ and $g_{33}$ values (50 volume percent PZT) of about $42 \mathrm{pC} / \mathrm{N}$ and $65 \mathrm{mVm} / \mathrm{N}$. These results compare favourably and show the highest $g_{33}$ value in comparison with other reported data in the literature. The experimental data for $\varepsilon_{\mathrm{r}}$ agree quite well with the Lichtenecker model for PZT volume fraction up to 50\%, while the Yamada and Jayasundere model underestimate the experimental data. The experimental data for the piezoelectric constants agree well with the Yamada model, suggesting an elongated particle shape and confirmed by independent image analysis. Moreover, the addition of PA11 to PZT/LCT composites leads to a lower elastic modulus providing more flexibility to the materials. From the above observations we conclude that these composites are suitable candidate for both sensors and may even show possibilities for actuators.

\section{Acknowledgements}

This work was financially supported by the Smartmix funding program (grant SMVA06071), as part of the program "Smart systems based on integrated Piezo". The authors are grateful to Morgan Electro Ceramics (Ruabon, United Kingdom) for providing the PZT powder used in this research and to Prof. Dr. Sybrand van der Zwaag and Dr. Theo Dingemans of Delft University of Technology for providing the LCT polymer used in this research.

\section{References}

[1] Furukawa T, Ishida K and Fukada E 1979 J. Appl. Phys. 50 4904-4912

[2] Dasgupta D K and Abdullah M J 1988 J. Mat. Sci. Letters 7 167-170

[3] Chan H L W, Chen Y and Choy C L 1996 Trans. Dielectrics and Electrical Insulation 3 800-805

[4] Akdogan E K, Allahverdi M and Safari A 2005 Trans. Ultrasonics, Ferroelectrics and Frequency control 52 746-775.

[5] Jaffe B, Cook Jr W R and Jaffe H 1971 Piezoelectric ceramics (New York) 49-50 
Highlighted Version

[6] Newnham R E, Skinner D P and Cross L E 1978 Mat. Res. Bull. 13 525-536

[7] Choi J, Hahn B, Ryu J, Yoon W, Lee B and Park D 2009 Sensors and Actuators A 153 89-95

[8] Wegener M and Arlt K 2008 J. Phys. D: Appl. Phys. 41 1-6

[9] Venkatragavaraj E, Satish B, Vinod P R and Vijaya M S 2001 J. Phys. D: Appl. Phys. 34 487-492

[10] Bai Y, Cheng Z Y, Bharti V, Xu H S and Zhang Q M 2000 Applied Physics Letters 76 3804-3806

[11] Knijnenberg A, Weiser E S, StClair T L, Mendes E and Dingemans T J 2006 Macromolecules 39 6936-6943

[12] Van den Ende DA, Almeida de P and Van der Zwaag S 2007 J. Mater. Sci. 42 6417-6425

[13] Amer A M, Ibrahim S A, Ramadan R M and Ahmed M S 2005 J. Electroceramics 14 273-281

[14] Zhang H, Uusimaki A, Leppavuori S and Karjalainen P 1994 J. Appl. Phys. 76 4294-4300

[15] Image J: http://rsbweb.nih.gov/ij/ version 1.42 q release 08-06-09, accessed 20-01-10

[16] Satish B, Sridevi K and Vijaya M S 2002 J. Phys. D: Appl. Phys. 35 2048-2050

[17] Sa-Gong G, Safari A, Jang S J and Newnham R E 1986 Ferroelectrics Lett. 5 131-142

[18] Yamada T, Udea T and Kitayama T 1982 J. Appl. Phys. 53 4328-4332

[19] Furukawa T, Ishida K, Fukada E 1979 J. Appl. Phys. 50 4904-4912

[20] Firmino Mendes S, Costa C M, Sencadas V, Serrado Nunes J, Costa P, grgorio jr. R, LancerosMéndez S, 2009 J. Appl. Phys. A 96 899-908

[21] Jayasundere N and Smith B V 1993 J. Appl. Phys. 5 2462-2466

[22] Khastgir D and Adachi K 1999 J. Polymer Science 37 3067-3070

[23] Lichtenecker K 1926 Physikalische Zeitschrift 27 115-158 
Highlighted Version

[24] Sihvola A 1999 Electromagnetic mixing formulas and applications (London) 166-167

[25] Sillars R W 1937 J. Inst Electr 80 378-394

[26] Zakri T, Laurent J-P, Vauclin M 1998 J. Phys. D 31 1589-1594

[27] Wong C K, Poon Y M, Shin F G 2001 J. Appl. Phys. 90 4690-4700

[28] Turik A V, Chernobabov A I, Radchenko G S, Turik S A 2004 Physics of the Solid State 46 22132216

[29] Bruggeman D A G 1935 Ann. Physik 24 636-664

[30] Reynolds J A, Hough J M 1957 Proc. Phys Soc. London B70 769-774

[31] Marutake M 1956 J. Phys Soc. Japan 11 807-814

[32] Piezoelectric Materials Data Book 1996 Ferroperm, Kvistgård, (Denmark) 
Dear Editor,

Our article, entitled "Processing and characterization of piezoelectric 0-3 PZT/LCT/PA composites" (Article ID: D/352960/PAP), was submitted to Journal of Physics D: Applied Physics on $3^{\text {rd }}$ April 2010. The editorial decision and reviewer's comments were returned to us on $4^{\text {th }}$ May 2010. In view of some extension of the work we asked for a somewhat longer response period, which you kindly granted, the deadline being July 30 .

We have addressed the reviewer's comments in detail in the attached addenda (Reply to reviewers). We have also amended the manuscript accordingly. Moreover we have highlighted the changes made in the revised manuscript.

We are grateful to you for reviewing our article and for the opportunity to submit the revised manuscript to Journal of Physics D: Applied Physics.

On behalf of my co-authors,

With kind regards,

Ir. Indu Babu. (Corresponding Author)

\author{
Ir. Indu Babu (Ph.D Student) \\ Laboratory of Materials and Interface Chemistry \\ Department of Chemical Engineering and Chemistry \\ Helix building, STO 2.28 \\ Eindhoven University of Technology \\ PO Box 513, 5600 MB Eindhoven, The Netherlands \\ Tel: +31 402473066 \\ Fax: +31 402445619 \\ i.babu@tue.nl
}


Highlighted Version

\section{Addendum: Reply to reviewers report 1: Referee 1}

First of all, we would like thank the reviewers for their comments and we address the various points raised in detail below.

1. The g coefficient was calculated by dividing $d$ on dielectric permittivity (DP). The authors claim, that it has the highest value in nature. However in the experiment DP was measured at $1000 \mathrm{~Hz}$, but $\mathrm{d} 33$ at $110 \mathrm{~Hz}$ by d33-meter. It is obvious, that it is impossible to measure these parameters at different frequencies and obtain $\mathrm{g}$ in such manner. At $110 \mathrm{~Hz}$ the DP will be much greater than the same for $1000 \mathrm{~Hz}$ due to the excluded mechanisms of polarization in the present study. So, according to my point of view, g-coefficient is calculated in a wrong way.

- We agree with the reviewer's comment that the parameters $\varepsilon_{\mathrm{r}}$ and $d_{33}$ should be measured at the same frequencies. Indeed we measured the dielectric permittivity $\varepsilon_{\mathrm{r}}$ and $d_{33}$ at two different frequencies. As expected (at least by us) measurement of the permittivity at 110 $\mathrm{Hz}$, the frequency used for $d_{33}$, showed no difference as compared to the value at 1000 $\mathrm{Hz}$. In fact in the mean time we have shown that $\varepsilon_{\mathrm{r}}$ remains the same over a range of frequencies as shown in figure 6 (b) in the revised version of the manuscript, showing the dependence of permittivity as a function of log frequency. From this figure it is observed that the DP remains the same throughout the frequency range. Hence the conclusion is not changed but the text is adapted accordingly.

2. The results of the paper are still meaningless without careful analysis of the elastic properties of obtained composite. The usage of very soft components, strong percolation of $d$ by $1100 \mathrm{C}$, and leakage of the initial components require sufficient mechanical tests before usage in technical devices. I recommend resonance method for measuring elastic compliance or stiffness.

- We agree with the reviewer's comment saying that results of the paper are incomplete without careful analysis of the elastic properties of obtained composite. The elastic modulus of the composites was tested in a three-point bending mode on a TA Instruments Q800 series DMA at room temperature. The obtained results are shown and discussed in section 3.2.4. Mechanical properties by DMA (revised version).

3. The theoretical analysis is done poorly. The Lichtenecker formalism is primitive for such task and cannot be applied, if the ratio between DP of components is more than 6-7. Here this ratio is equal to at least $\mathbf{1 0 0 0}$. It is also impossible to analyze pure dielectric properties without taking into account piezoelectric and elastic interactions (see, for example, Physics of the Solid State, Vol. 46, No. 12, 2004, pp. 2213-2216). The Yamada's model is almost unknown for the scientific community and was developed in 1982 year (28 years before this article). Such old models are rarely to use nowadays. I recommend to the authors to find 
more up-to-date literature and describe the model correctly including the description of the clamping effect by piezomeasurements.

- The reviewer states that the Lichtenecker formalism is not applicable if the ratio between the components is higher than 6-7 but does not back this argument with a reference or argument. One could argue that this manuscript proves that the Lichtenecker formula is suitable for higher ratios. However, there is more proof (see A. Sihvola's book: Electromagnetic mixing formulas and applications, ISBN 0852967721) where Lichtenecker's rule is applied and shown to hold for a ratio of 16. Moreover, some 10 years ago, a theoretical backing proof of the Lichtenecker rule was given based on effective medium theory without any restriction on permittivity ratio (J. Phys. D 31(1998)1598). Based amongst others on this paper the authors believe that the Lichtenecker rule is theoretically credible and therefore perfectly suitable as a rule of mixtures for describing the properties of the composites found in this work. Accordingly the arguments to use this model have been sharpened and incorporated in the revised text.

- The article Physics of the Solid State 46(2004) 2213-2216 (as quoted by the reviewer), has no experimental backing at all and deals with an entirely different frequency range. Moreover, increased conductivity was used leading to probably unacceptable tan delta levels (not stated in that manuscript). It is evident from results of dielectric measurements presented in this paper that there is no reason to believe that any "giant response" should occur at all in this frequency range, especially since this kind of response has never actually been witnessed before. A brief discussion is introduced referring to this paper.

- In response to the reviewer's statement that "the Yamada's model is almost unknown for the scientific community", the authors beg to differ because we believe this is a very relevant model. To back this view we can argue that Yamada's article has been cited no less than 90 times in Scopus (search date: 2010-06-17) by articles appearing in journals such as Appl. Phys. Lett., J. Phys. D, J. Appl. Phys. and Appl. Phys. A. It was used as the primary model describing the dielectric properties of PMN-PT powder-polymer composites in Appl. Phys. Lett. 76, 3804 (2000), a highly cited article (>180 citations in Scopus since 2000). We therefore believe the statement mentioned does not hold. Furthermore, we are puzzled by the argument that a model should be disregarded because it is "old". Yamada's article which describes the model continues to be used (cited 48 times since 2005). We introduced a brief section on theory discussing a few of the most important models used.

\section{The authors ought to interpret the results obtained in the frame of percolation theory and creation of the cluster by calcination.}

- The data were analysed in the frame of other than percolation models. For discrepancies at higher filling fractions percolation may play indeed a role. However, a clear percolation threshold is not observed experimentally and therefore seems to play a minor role. On the other hand, the creation of clusters (we assume the reviewer means 
agglomerated PZT particles) during calcination does occur and a few remarks about their presence are made in the (original and revised) manuscript.

\section{The figure 9 contains one theoretical and two experimental curves. For which of them the model was applied?}

- We assume that the reviewer means one line and two sets of data points from the $d_{33}$ measurements for both composites (PZT/LCT/PA and PZT/PA). They are both compared with the Yamada model but since the contribution of the polymer is small anyhow, the prediction is mainly dependent in the volume fraction. The text has been changed accordingly.

6. All the literature is very old and not up-to-date. It has to be rewritten by new actual references.

- We don't agree with the reviewer's comment saying that all the literature is very old and not up-to-date. In order to correlate the performance behavior (permittivity and piezoelectric properties) of a diphase system like 0-3 composites, the mostly used models were from 1904 onwards. Maxwell Garnett developed the first analytic expression to predict the permittivity of a diphase system and the equation is still widely used. (See, for example, Firmino Mendes S et al., 2009, J. Appl. Phys. A, 96, page 900). As said in the response to point 3 , a brief section on theory discussing a few of the most important models is introduced.

7. The data about the parameters of the used components ought to be in a separate table. I recommend to the authors to add also the conductivity of components.

- We agree with the reviewer that a separate table of data used is helpful and hence the data about the parameters of the components used are now given in table 2. The dc conductivity of the PZT/PA and PZT/LCT/PA composites is also measured and given in this table. Moreover, we introduced the frequency dependent conductivity in section 3.1.3. Impedance data. The text is adapted accordingly. 


\section{Reply to reviewers report 2: Referee 2}

1. Page 2. In the introduction section, the authors state that "To overcome this limitation and to process fairly flexible composites, we developed a new series of 0-3 piezoelectric composites (PZT/LCT/PA) by incorporating PZT5A4 into a matrix of LCT and polyamide (PA-11)'. The authors didn't mention in the paper why/how the newly developed material PZT/LCT/PA overcomes the limitation of brittle property of PZT/LCT. Are there previous researches on PZT/LCT/PA in the literature? In the paper, the authors present the comparison of relative permittivity, piezoelectric charge constant, piezoelectric voltage coefficient of LCT/0.4PZT, LCT/PA/0.5PZT, and other piezoelectric materials in Table 2. Is the paper aimed to develop a material with high permittivity and piezoelectric constant? Please clarify the background and motivation of current work.

- We agree with the reviewer's comment saying that we didn't sufficiently mention in the paper why/how the newly developed material PZT/LCT/PA overcomes the limitation of brittle property of PZT/LCT. The answer to the question why we mentioned the newly developed material PZT/LCT/PA overcomes the limitation of brittle property of PZT/LCT is because we think that the addition of $20 \%$ PA will impart some degree of ductility to the present composite, as the elastic modulus is significantly lower. Here we refer to the response to point 2 of reviewer 1 . In the revised version elastic data have been added, although strength measurements are not done so far.

- A study on PZT/LCT composites was already done by van den Ende et al. (Van den Ende DA, Almeida de P and Van der Zwaag S 2007 J. Mater. Sci. 42 6417-6425) and this paper is referenced in the revised version of the manuscript.

- The goal of the present study is indeed to develop a new composite with better permittivity and piezoelectric constant. The introductory text has been changed accordingly.

2) Page 8. The authors say that "The maximum relative permittivity measured for the PZT/PA composites with different calcinations temperature is 32 for the PZT calcined at $1100{ }^{\circ} \mathrm{C}$." Figure 4 shows that the maximum relative permittivity occurred at both $1000{ }^{\circ} \mathrm{C}$ and $1100{ }^{\circ} \mathrm{C}$. Additionally, Figure 4 also shows that tan $\delta$ is not nearly "constant" in the temperature range of 800 to $1300{ }^{\circ} \mathrm{C}$. What is common range of loss tangent? Please check about the statement in this paragraph.

- We thank the reviewer for the constructive comments. It is true that the maximum relative permittivity occurred at both $1000{ }^{\circ} \mathrm{C}$ and $1100{ }^{\circ} \mathrm{C}$ in the graph but other reasons are indicated to prefer the data at $1100{ }^{\circ} \mathrm{C}$ above those at $1000{ }^{\circ} \mathrm{C}$. Considering that $\mathrm{X}$ ray diffraction showed that the PZT calcined at $1100{ }^{\circ} \mathrm{C}$ shows a maximum intensity of the tetragonal perovskite peaks at $(200) \mathrm{T}$ and $(211) \mathrm{T}$, indicating that at this temperature the material has become (XRD) single phase, it is concluded that PZT calcined at 1100 ${ }^{\circ} \mathrm{C}$ is the best choice. Nevertheless, based on permittivity data only, $1000{ }^{\circ} \mathrm{C}$ and $1100{ }^{\circ} \mathrm{C}$ 
are equally 'good'. The text has been changed accordingly. For tan $\delta$ nearly constant means ranging from 2 to $4 \%$. In order to recheck these data we conducted the impedance measurements at room temperature in a frequency range of $50 \mathrm{~Hz}-5 \mathrm{MHz}$ and the results are presented in the section 3.1.3. Relative permittivity and loss tangent. In order to prevent the doubling of the results we excluded the previous results. The text has been changed accordingly.

3. Page 11-13. The authors mentioned theoretical data and models developed by other researchers. Jayasundere, Yamada, and Lichtenecker models have been used in comparison. It will be better if theoretical equations/formulas of other models are presented in this paper. Alternatively, the authors can also briefly explain the differences of these models. Have the tests perfectly performed to generate accurate results or are the theoretical models more accurate? It looks like that the authors use current experimental results as the standard to judge the performances of theoretical models.

- According to reviewer's comment we included a new part (Section 2.4. Theory) to explain the analytic expressions that are mostly used for 0-3 composites and also briefly explained the differences between these models. In this study, we compared the obtained experimental results with Yamada, Jayasundere, and Lichtenecker models both for dielectric and piezoelectric constants to observe the correlation between the experimental values and theoretical models. We use the theoretical models to assess the performance of our new materials. The text is adapted accordingly.

4. Page 14. The reviewer is not clear about the usage of Section 3.2.4 in the paper? Have the authors tried to present the difference of PZT/LCT/PA composites with $20,30,40,50$, and 60 vol\% PZT using SEM morphology? Or intend to say PZT particles of these composites are well attached to polymer matrix? Are there any significant differences between the composites with 20, 30, 40, and 50\% PZT and the composite with 60 vol\% PZT? Please clarify this point.

- We agree with the reviewer's comment saying that the section 3.2.4. (SEM morphology) is not clear. In this section we would like to show the similarity of the morphology of the 20, 30, 40 and $50 \mathrm{vol} \%$ PZT. The larger magnification for $60 \mathrm{vol} \% \mathrm{PZT}$ is to show homogeneity on the micrometer scale while the detailed view is to show the adhesion of ceramic particles to the polymer matrix in $60 \mathrm{vol} \%$ PZT. By presenting this we would like to convey that the quality of the composites depends on the homogeneous distribution of the ceramic particles in the polymer matrix which correlates with its effectiveness for improving the properties of the composites.

- So, in order to make it clear, we showed all the figures together and marked the last figure as detailed view of the $60 \mathrm{vol} \%$ PZT. The text is changed accordingly. 
Dear Editor,

Our article, entitled "Processing and characterization of piezoelectric 0-3 PZT/LCT/PA composites" (Article ID: D/352960/PAP), was submitted to Journal of Physics D: Applied Physics on $3^{\text {rd }}$ April 2010. The editorial decision and reviewer's comments were returned to us on $4^{\text {th }}$ May 2010.

Revised manuscript was submitted on $22^{\text {nd }}$ July 2010 and the editorial decision was returned to us on $20^{\text {th }}$ August 2010.

I have addressed the reviewer comments in detail in the attached addenda. I have also amended the manuscript accordingly. I have highlighted the changes made in the revised manuscript.

We are grateful to you for reviewing our article and for the opportunity to submit the revised manuscript to Journal of Physics D: Applied Physics.

On behalf of my co-authors,

With kind regards,

Ir. Indu Babu. (Corresponding Author)

Ir. Indu Babu (Ph.D Student)

Laboratory of Materials and Interface Chemistry

Department of Chemical Engineering and Chemistry

Helix building, STO 2.28

Eindhoven University of Technology

PO Box 513, 5600 MB Eindhoven, The Netherlands

Tel: +31 402473066

Fax: +31 402445619

i.babu@tue.nl 


\section{Addendum:}

First of all, we would like thank the reviewer for the comments and we address the various points raised in detail below.

[1] What do authors mean by d10, d50 and d90 in table 1 on page 9?

- The label $d_{10}, d_{50}$ and $d_{90}$ stands for the undersize percentage of the cumulative particle size distribution. On page 8 the text has been changed accordingly.

[2]. At what frequency d33 and g33 was measured (figures 5 and 7; g33=d33/\&0er (equation.1))? Both $\mathrm{d} 33$ and relative permittivity (\&r) are frequency dependent. Hence, either both these parameters must be constant in a given frequency range or the measurement should be done at the same frequency. Nowhere in the graph or text there has been a mention about the frequency at which g33 parameter was evaluated.

- Since the frequency of the $d_{33}$ meter is fixed at $110 \mathrm{~Hz}$, the value of the relative permittivity $\varepsilon_{\mathrm{r}}$ is also taken at the same frequency to calculate $g_{33}$. On page 5 as well as in the legends of figures 5 and 7 the text has been changed accordingly. Since the frequencies had to be the same, we now used impedance analysis which changed the permittivity values for the 40, 50 and $60 \%$ marginally.

[3]. In subsection 3.2.1- 'Impedance data': What could be reason for increase in conductivity as the volume fraction of PZT increases?

- Because interface conductivity is usually higher then bulk conductivity, the increase in the conductivity with the increase in volume fraction of PZT is attributed to the increased contribution of interface conductivity, directly related to the increasing particle volume fraction. On page 11 this text is added.

[4]. There is a typographical error on page \# 12 “... A maximum value for $\mathrm{d} 33$ of $46 \mathrm{C} / \mathrm{N}$ is observed for 60 volume percent PZT." --> it should be 46 pC/N.

- Indeed. On (now) page 13 the maximum value for $d_{33}$ is changed to $46 \mathrm{pC} / \mathrm{N}$.

[5]. In section 2.4 "Theory": some symbols are defined multiple number of times, e.g. \&c , \&p , etc. this should be corrected.

- In section 2.4. Theory, the repetitions in $\varepsilon_{\mathrm{c}}$ and $\varepsilon_{\mathrm{p}}$ are removed. Only the highlighted phrase is kept.

[6]. The authors must incorporate a detailed explanation related to ananlysis of their results in the frame work of percolation theory and creation of cluster by calcinations: Using percolation theory, one can observe whether percolation threshold is reached or not. How can the authors conclude by writing - 'it is clear that percolation threshold is not observed' and that' percolation theory plays a minor role'. A detailed explanation is required.

- This is complex point but a short paragraph discussing the results in the light of percolation is added in section 3.2.3. 